\title{
Improving of the water preparation systems in the industry thermal power plants
}

\author{
Daniela Choshnova ${ }^{1, *}$ \\ ${ }^{1}$ UCTM, Kliment Ohridski blvd.8, Sofia, 1756 Bulgaria
}

\begin{abstract}
Some modern possibilities for modernization of the water preparation in the heat power plants of metallurgical enterprises are presented. It is focused on the ecological and technological advantages of the methods for obtaining of ultra-pure water. This work is not intended to justify the need of usage of particular technology in an object. This is a separate task requiring in-depth consideration.
\end{abstract}

\section{Introduction}

Natural water can not be used in modern technological processes without pre-treatment. It needs to be softened and demineralized by ion exchange treatment in the industrial unit in heat power plants. In this way the necessary quality of the water circulating in the energy boilers is achieved, also the produced steam for technological needs is achieved. Some negative phenomena are preventing. They are causing:

- deposition of salts on the heating surfaces of the steam generators;

- deposition of salts on the blades, nozzles and guides of the steam turbines, which creates a risk of imbalance of the impeller, narrowing of the space between the blades, which leads to decreasing of the aggregates power;

- decreasing the flow rate of the steam from steam generators, respectively their productivity;

- creating conditions for overheating the heat exchange surfaces of the steam generators, etc ;

Despite the extensive researches in creating new generations resins and their usage in the existing ion-exchange installations, there are some significant drawbacks. For example, low hydrophilicity, which result in low diffusion rates of ions inside and low sorption rates and desorption. In practice, they are used in granular form whose bonding in the ion exchange equipment during the sorption process leads to the need for forced rupture and their gradual mechanical degradation during the operation. In addition, they require frequent regeneration to restore their ion-exchange capability.

Special procedures are required for the preparation of regeneration solutions, their storage and the protection of the personnel against possible leaks. The cost of reagents, as a rule, exceeds 2-3 times the stoichiometric quantities. Accordingly as many times, waste products (strongly acidic and strongly alkaline industrial waters) are increased, which must

\footnotetext{
*Corresponding author: daniela@uctm.edu
} 
be neutralized before being discharged into the sewerage system. In the end, all this is reflected in large capital and operating costs.

For solving the above-mentioned technological problems, if possible, a series of apparatuses or their modified models are used, but recently they are created with original constructions for a particular process. A large number of variants for countercurrent regeneration with different ionites have been investigated, and according to the authors [1], with industrial applications are those in which a continuous layer is provided.

Article [2] presents a method for improving the control during regeneration of the filters in a chemical water treatment section in a heat power plant of a metallurgical plant. It reduces the consumption of sulfuric acid and sodium hydroxide and allows high-quality desalted water to be produced by minimizing the side unfavourable processes of steam generators.

The report in [3] is dedicated to the advantage of conjugated regeneration, which is an inexpensive option for decreasing reagent costs and reducing the waste water pollution. In [4] are presented results from comparative study of different schemes for combination of membrane and ion exchange technologies applied in Russian heat power plants. The impact of various factors (composition of water, type of equipment, etc.) on the sustainability of the end-product indicators is examined. Detailed analysis of reagent and power consumption for each installation is given.

In general, a number of complementary factors - reliability, environmental friendliness, capital and operating costs - have to be respected when choosing a water conditioning modernization scheme. The literature presents models that allow to evaluate other external factors that influence the efficient operation of ion-exchange treatment of water - the flow composition; a pre-cleaning method, etc. Separate publications on comparing the performance of different technologies, though fairly objective, carry the characteristics of a particular case and relate to the specific operating conditions.

This work is not intended to justify the need to use a particular technology in an object. This is a separate task requiring in-depth consideration. Here it is resented some modern opportunities for modernization of water treatment in the heat power plants of industrial enterprises.

\section{Technological improvement of existing water treatment systems in industrial thermal power plants}

The requirements for the quality of the treated water in the chemical treatment facilities for the heat power plants are shown in Table 1.

Table 1. Requirements for the quality of feed water for steam generators in industrial heat power plants

\begin{tabular}{|l|c|}
\hline Parameter & Permissible norm \\
\hline Specific conductivity & $<0,2 \mu \mathrm{Sm} / \mathrm{cm}$ \\
\hline Hardness & $<5 \mathrm{mg}$-eq $/ l$ \\
\hline Sodium concentration & $3-10 \mathrm{mg} / l$ \\
\hline Silicone acid concentration & $<0,2 \mathrm{mg} / l$ \\
\hline Concentration of chlorides & $<3 \mathrm{mg} / l$ \\
\hline Concentration of organic carbon & $100-300 \mathrm{mg} / l$ \\
\hline Concentration of sulfates & $<3 \mathrm{mg} / l$ \\
\hline
\end{tabular}

Conventional ion exchange systems shown in Fig.1 are not as complicated as construction and are reliable in operation. However, as mentioned, all their disadvantages are related to the need to use large quantities of ionic mass recovery reagents. Regenerative 
solutions are aggressive and contain components that go beyond environmental standards, and the process itself is not automated.

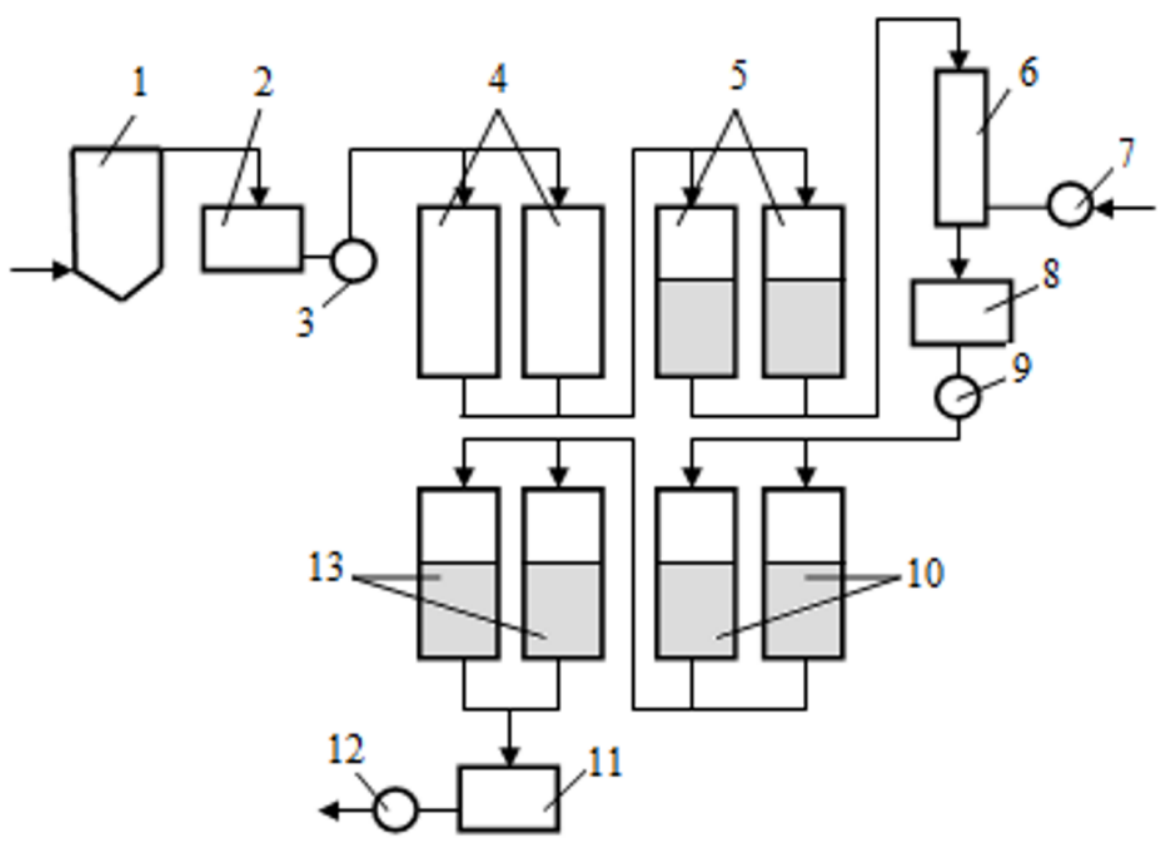

Fig. 1. Conventional ion-exchange installation: 1 - reactor-precipitator; 2 - clear water tank; 3 - clear water pump; 4 - sand filters; 5 - strong cationite filters; 6 - deaerator; 7 - air fan; 8 - cationic water tank; 9 - cationic water pump; 10 - weak anionic filters; 11 - demineralized water tank; - 12 demineralized water pump; 13 - strong anionic filters

In some heat power plants these acidic and alkaline waters are mutually neutralized in the tanks in which they are mixed. Often, however, in the ion exchange plants the regenerations of the two types of filters do not take place simultaneously. In addition, sulfuric acid streams contain larger amounts of acid than the sodium hydroxide in the alkaline. For this reason, it is necessary to neutralize the waste water, which is collected in a common vessel and treated with a reagent [3]. A stock of chemicals is also needed, which is accompanied by additional costs.

In this connection, there is a need for increased control and monitoring of spent water, improvement of existing technologies or replacement with new more efficient ones.

Modern alternatives for ion exchange are ultrafiltration using mix-bad filters and compact layer membrane technologies including reverse osmosis and / or electrodeionization. They allow to improve the quality of treated water with minimal reagent consumption.

\subsection{Installation of mix-bed filters after the main scheme}

In Fig. 2 is a modernization scheme comprising installing mix-bed filters following the basic ion exchange treatment scheme. Deep downstream processing in them allows to achieve a specific conductivity below $0.3 \mu \mathrm{S} / \mathrm{cm}$ and a silicate content of less than $0.02 \mathrm{ppm}$. 


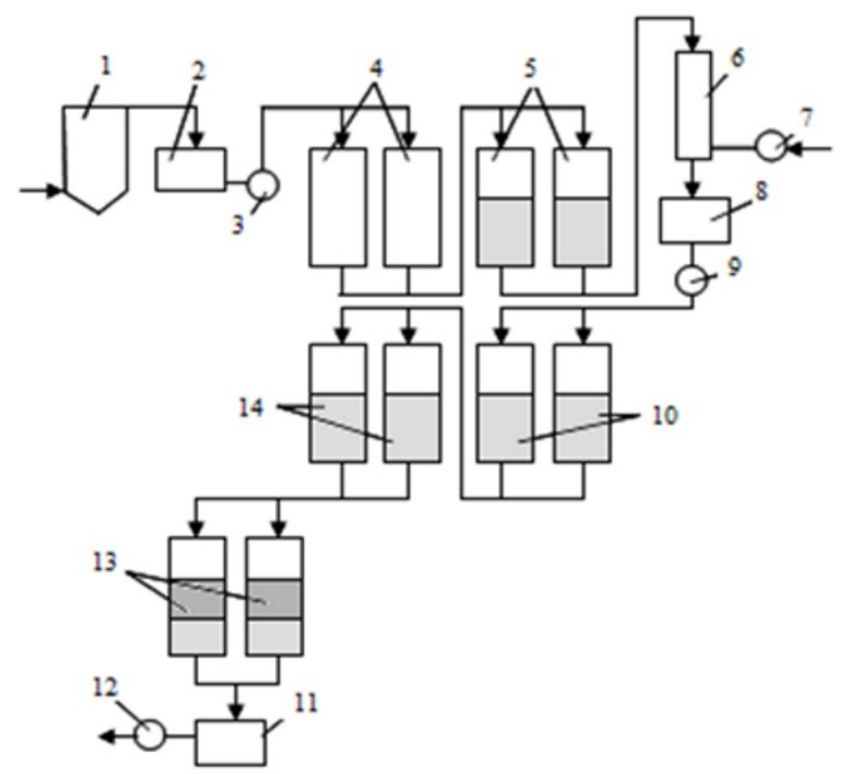

Fig. 2. Ion-exchange installation with purification of the technological flow in mix-bed filters: 1 reactor-precipitator; 2 - clear water tank; 3 - clear water pump; 4 - sand filters; 5 - strong cationite filters; 6 - deaerator; 7 - air fan; 8 - cationic water tank; 9 - cationic water pump; 10 - weak anionic filter; 11 - ultra demineralized water tanks; 12 - ultra demineralized water pump; 13 - mix-bed filters; 14 - strong anionic filters

\subsection{Final purification by electro-deionization of the stream}

The electro-deionization is a highly efficient technology for ultrafiltration with $90-95 \%$ efficiency and features: constant quality of the filtrate, obtaining of ultrapure water with conductivity below $0.1 \mu \mathrm{S} / \mathrm{cm}$, silica content below $0.02 \mathrm{ppm}$. The process is continuous and fully automated. The system is operated with a constant electric field and consists of ionselective membranes located in a compact unit which is installed after the main scheme of the ion exchange system as shown in Fig.3. 


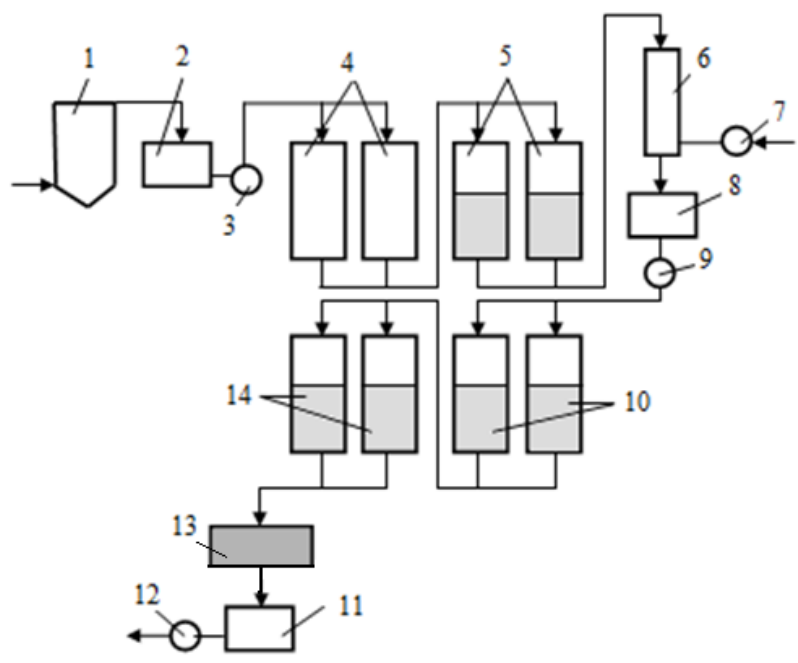

Fig. 3. Scheme of an ion-exchange installation with purification of the process stream by electrodeionization: 1 - reactor-precipitator; 2 - clear water tank; 3 - clear water pump; 4 - sand filters; 5 strong cationite filters; 6 - deaerator; 7 - air fan; 8-cationic water tank; 9 - cationic water pump; 10 weak anionic filter; 11 - ultra demineralized water tanks; 12 - ultra demineralized water pump; 13 electro-deionization system; 14 - strong anionic filters

\subsection{Combined installations with reverse osmosis membranes}

Water purification by reverse osmosis systems involves the forced passage of the treated water through a semipermeable membrane. In most cases they are not universal. Typically, the scheme is developed taking into account a number of factors, including the business itself, the required volumes of purified water, and so on [6].

High purified water can be obtained by two-stage osmosis, in which the water stream is purified from salts to $97 \%$ and a specific electrical conductivity of $1-5 \mu \mathrm{Sm} / \mathrm{cm}$ before final purification in mix-bed filters or electro-deionization module (Fig.4).

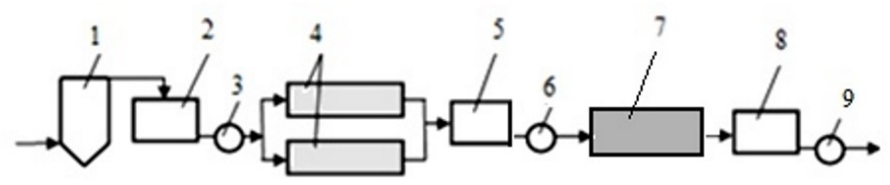

Fig.4. Scheme of an ion-exchange installation with reverse osmosis combined with electrodeionization system or mix-bed filters: 1 - reactor-precipitator; 2 - clear water tank; 3 - clear water pump; 4 - reverse osmosis membranes; 5 - partial desalinated water tank; 6 - partial desalinated water pump; 7 - electro-deionization system or mix-bed filter; 8 - ultra demineralized water tank; 9 - ultra demineralized water pump

The advantages of membrane technologies based on reverse osmosis have been thoroughly studied. In $[4,5]$ there are models for optimizing the consumption of chemicals and labor by adding a reverse osmosis module to existing installations. In [4] are presented 4 variants of a combined scheme, which are applied to a water flow with different mineralization. According to expert data, if raw water is highly mineralized and there is an 
increased consumption of sodium, a technological solution is the use of membrane technologies including reverse osmosis and purification by electro-deionization. But with increased electricity consumption and low-mineralized raw water the technological solution is reverse osmosis and mix bed filters. When the salt content in the raw water is variable, the second scheme is preferable.

\section{Analysis of the different options}

Conventional ion-exchange water treatment schemes for technological purposes are used in systems with high productivity and low salt content of raw water. When changing its composition and the requirements for steam production, the choice of a suitable method for its improvement is influenced by the following factors:

- expenditure of treated water in the power plant as a whole;

- way of removing and neutralizing waste streams;

- value of reagents for recovery of ion-exchange resins (mainly sodium base), incl. transport and storage costs;

Electro-deionization (EDI) is the newest method that can be successfully applied to heat power plants of industrial plants. Despite the high cost of equipment, it has some technological advantages that can not to be ignored:

- at low salt content of the water flow in the installation does not require preliminary treatment;

- uninterrupted work, with no need to use chemicals;

- it maintains constant water conductivity below $0.1 \mu \mathrm{S} / \mathrm{cm}$;

- it requires low energy consumption for system performance and less labor input;

- equipment compactness;

- complete automation and full control of the process;

- safety for personnel and the environment.

The advantages of the EDI system to mix-bed filters are:

- there is no cost for regeneration chemicals and water for deionization and recovery;

- no waste treatment costs, incl. building special equipment for this;

- complete automation and process continuity.

According to the data from the practice, in a heat power plant at a metallurgical plant for the production of non-ferrous metals in our country, the costs for construction of the schemes presented in Figs. 2 and 3 are comparable (at the same flow rate of treated water). But in Fig. 2 contaminated waters are eliminated, neutralization of which requires the construction of neutralization facilities. Furthermore, as mentioned, costs for acid and base for regeneration of resins in mix bed filters, their servicing and maintenance are needed.

The problem of ensuring the fault-free operation of the installation comprising an electrodeionization module consists in its sensitivity to all sorts of disturbances in the operation of the precedent filters. This can leads to a deterioration in the quality of the water supplied to it. A basic requirement is that the residual iron content in inlet EDI modules water is below $0.01 \mathrm{ppm}$, because it blocks the ion-exchange effect of the resins in it. Once deposited in them it isn't possible to be removed. When it is used in combination with reverse osmosis membranes and a highly mineralized primary stream, there is a possibility of accumulation of organic and mineral deposits polluting the intermembered spaces in the individual elements.

The combined schemes shown in Figs. 2-4 can be used for the reconstruction of existing installations where ion-exchange filters, collection and neutralization systems already exist. In their implementation, they will decrease, respectively eliminates (in variant reverse osmosis-electro deionization) the amount of waste streams and the reagent consumption. 
The main components of industrial reverse osmosis systems include pre-mechanical water purification filters and a high pressure pump prior feeding the membranes. Due to the probability of its contamination, a dispenser for anti-caking agents and biocides should also be included to prevent organic deposition.

The economic efficiency of water treatment using membrane technologies is substantially higher than conventional ion-exchange installations. Significantly increased operational reliability, lower labor costs and reagents, and the role of human factor due to automation of the process.

For the successful realization of projects for modernization of ion exchange systems, it is necessary to choose the right equipment (pumps, valves and apparatus, control and measuring devices).

\section{Conclusion}

Obtaining of ultra-demineralized water in power plants is one of the main tasks in steam production for technological needs. Although the ion exchange installations have been in operation for a long time, their modernization is necessary. This will leads to decreasing of capital and operating costs increasing of the ecological efficiency, incl.

- decreasing the technological time for putting the equipment into operation;

- reduction of the environmental risk in servicing the waste streams neutralization system;

- decreasing the cost of resin regeneration reagents of the filters;

- improvement of working conditions;

- savings for servicing and repair due to automation of the process.

The presented article would be useful for designing of new or for reconstruction of existing schemes for water preparation.

\section{Referenses}

1. B. Riabchikov, A. Sibirev et al., J. En. and Wat. Prep. 5, 43 (2006).

2. D. Choshnova, J. Sci. Proc. XXIV 1, 187, 104 (2016).

3. D. Choshnova, J. Sci. Proc. XXIV 1, 187, 107 (2016).

4. P. Surdilovski, N. Noev, P. Morin, J. En. and Wat. Prep. 2, 58 (2009).

5. A. Nikitenko, J. En. and Wat. Prep. 5, 61 (2009).

6. http://www.engineering-review.bg/ 